\title{
O uso da ferramenta computacional Scilab no ensino de raciocínio lógico-matemático para alunos de ensino médio
}

\author{
Tomaz, M. R. C. ${ }^{1 *}$; Da Silva, W. B. ${ }^{2} ;$ Dutra, J. C. S. ${ }^{2}$ \\ 1 Graduação em Engenharia Química, Universidade Federal do Espírito Santo, Alegre, ES, Brasil. \\ 2 Programa de Pós-graduação em Engenharia Química, Universidade Federal do Espírito Santo, Alegre, ES, Brasil. \\ *e-mail:mr-tomaz@hotmail.com
}

\begin{abstract}
Resumo
O uso de diferentes ferramentas de ensino se torna cada vez mais viável frente à evolução tecnológica atual. $\mathrm{O}$ ensino e aprendizagem de conteúdos relacionados a programação surge como uma alternativa quando se deseja desenvolver raciocínio lógico, apesar de ser recebido com certa dificuldade por parte dos discentes. O projeto apresentado neste artigo baseia-se no desenvolvimento do raciocínio lógicomatemático em alunos do ensino médio por meio do uso da ferramenta computacional Scilab. Notou-se que implementação de uma técnica de ensino diferente da usual contribui para o aprendizado, havendo maior assimilação de conteúdos teóricos pelos alunos, além de contribuir com a sua inserção em uma área que, normalmente, somente é vista no ensino superior.
\end{abstract}

\begin{abstract}
The use of different teaching tools becomes increasingly viable in the face of current technological developments. The teaching and learning of content related to programming emerges as an alternative when it is desired to develop logical reasoning, although it is received with some difficulty on the part of the students. The project presented in this article is based on the development of logical-mathematical reasoning in high school students through the use of the Scilab computational tool. It was noted that the implementation of a teaching technique different from the usual one contributes to the learning, having a greater assimilation of theoretical contents by the students, besides contributing with its insertion in an area that is normally only seen in higher education.
\end{abstract}

Keywords (Palavras chaves): Scilab, programming, reasoning.

\section{Introdução}

A programação, segundo Santos e Costa (2005), é definida como "arte ou técnica de construir ou formular algoritmos de uma maneira sistemática", sendo os algoritmos códigos escritos na linguagem de interesse a ser programada. No ensino superior, vários cursos das áreas tecnológicas contam com disciplinas que envolvem raciocínio lógico e se tem observado que é crescente a preocupação, por parte de docentes, com o processo de ensino e aprendizagem de conteúdos de programação (ROCHA et al., 2010). Por tratar de problemas que envolvem estratégias de solução com base lógico-matemática, a programação é recebida, por parte dos alunos, como algo abstrato e distante do campo de utilização. Este fato faz com que seja exigido do professor uma estratégia de ensino eficaz que acompanhe, atenda e avalie os alunos, por vezes não efetiva devido aos problemas, como por exemplo o volume de discentes por classe e diferentes níveis de aprendizado (RAABE; SILVA, 2005).

Para o ensino médio, as Diretrizes Curriculares do MEC estabelecidas pela Resolucão CEB № 3 pregam que medidas metodológicas de ensino sejam diversificadas, a fim de estimular a reconstrução do conhecimento e mobilizar o raciocínio (MEC,1998). 
Deste modo, o uso de ferramentas gráficas, animações e softwares surgem como ponto positivo no ensino lógico-matemático, auxiliando alunos que futuramente sigam em áreas que explorem esse domínio de conhecimento (SANTOS; COSTA, 2005). Várias pesquisas têm sido realizadas nesta área, sendo comprovado que a multidisciplinaridade contribui para o desenvolvimento do raciocínio lógico dos envolvidos (RAABE; SILVA, 2005).

Neste contexto, é apresentado no trabalho o funcionamento de um projeto desenvolvido com alunos do ensino médio que utiliza a ferramenta Scilab, software livre e de código aberto para computação numérica, a fim de desenvolver o senso lógico a partir de técnicas de programação e conhecimentos adquiridos pelos tutores no ensino superior.

\section{Metodologia}

\subsection{0 projeto}

O projeto desenvolvido pela parceria Universidade e Escola Pública se consiste na introdução do software Scilab (versão 5.2.2) aos alunos, com a apresentação teórica de toda a lógica de programação, e desenvolvimento de algoritmos que solucionem problemas vistos em áreas exatas, como matemática e física básicas. O objetivo principal está na contribuição para o desenvolvimento da base lógica-matemática dos alunos, visando auxiliar no processo de aquisição de novos conhecimentos.

\subsection{Plano de atuação}

Por se tratar de um projeto com um extenso plano de atuação, este foi dividido em semestres, a fim de melhor organizar o processo de ensino e aprendizagem do conteúdo ministrado.

A programação deve ser ensinada englobando conteúdos essenciais, como a introdução de linguagem de programação, conceitos, princípios e modelos (SANTOS; COSTA, 2005). Logo, a primeira etapa do projeto se baseou neste fato. Posteriormente, foram contextualizados e aplicados os conteúdos vistos a fim de capacitar os alunos a escreverem seus próprios algoritmos, partindo deles a interpretação de problemas matemáticos e físicos expostos.
2.3 O software e os códigos computacionais desenvolvidos

Várias tecnologias têm sido utilizadas no processo de ensino em diferentes níveis de educação, sendo os softwares educativos uma delas. Estes podem ser divididos segundo categorias e modalidades, sendo modelagem e simulação uma delas (DIM; ROCHA, 2011). O software Scilab fornece um ambiente computacional de trabalho que pode ser aplicado em áreas científicas, possuindo em seu banco de dados diversas funções matemáticas.

Aos alunos, em um momento inicial, foi apresentada toda a interface do programa e explicado todo seu funcionamento. A partir da ambientação destes à ferramenta, os algoritmos foram implementados.

Alguns dos algoritmos desenvolvidos envolviam calorimetria, movimento uniforme e uniformemente acelerado, criação e alocação de vetores, coeficientes linear e angular e velocidade média. Estes, sempre contextualizados em problemas de vestibulares. Como exemplo, tem-se o exercício 1 que foi implementado.

Exercício 1: Lançada em 1977, a sonda espacial Voyager 1 está, atualmente, a $1,5.10^{10} \mathrm{~km}$ da Terra. Suponha que, dessa distância, a Voyager 1 envie, para a Terra, um sinal de rádio que se propaga à velocidade da luz, que é de $300.000 \mathrm{~km} / \mathrm{s}$. Calcule o tempo, em horas, necessário para que o sinal chegue à Terra.

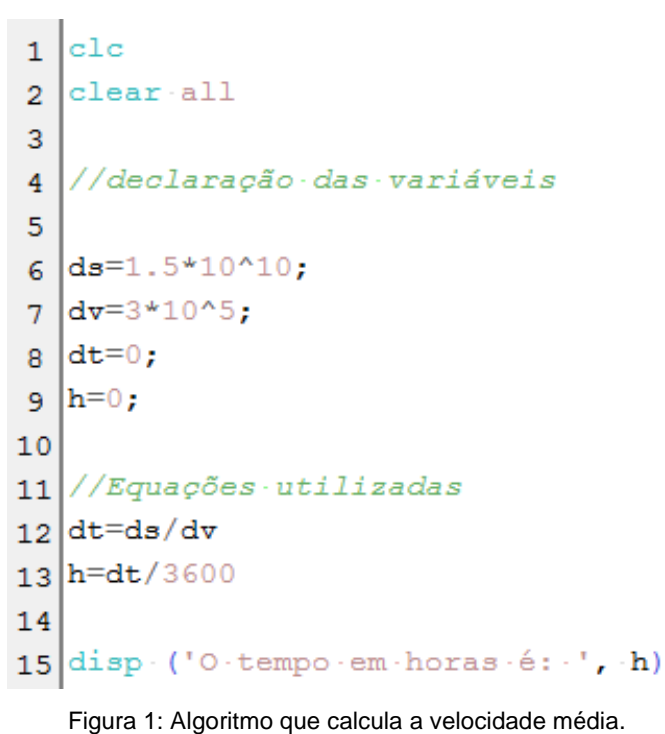

Figura 1: Algoritmo que calcula a velocidade média. 


\section{Encontro Científico de Física Aplicada}

\section{Blucher}

2.4 Equacionamento utilizado em algoritmos desenvolvidos

Foram tomadas equações básicas de matemática e física a fim de se implementar os algoritmos no software utilizado. Como um grande volume de exercícios foi realizado, cita-se unicamente como exemplo a equação empregada no algoritmo descrito na Figura 1. Para o cálculo de velocidade média, a equação 1 foi utilizada, onde $V_{m}$ corresponde a velocidade média, $\Delta S$ a variação da distância percorrida e $\Delta$ t o intervalo de tempo gasto.

$$
V_{m}=\frac{\Delta S}{\Delta t}
$$

\section{Resultados e discussão}

Ao longo do processo de ensino os alunos tiveram que lidar com um volume de informações que, em sua maioria, não haviam antes tido contato. Conteúdos básicos de programação, conceitos importantes da área e desenvolvimento de algoritmos foram etapas essenciais que possibilitaram o desenvolvimento de códigos computacionais que auxiliassem na trajetória acadêmica.

O raciocínio lógico-matemático foi desenvolvido a partir de um plano de ensino que objetivou entender as dificuldades individuais de cada aluno e auxiliar do melhor modo possível no melhoramento de seu desempenho. A utilização de vários métodos didáticos por parte dos monitores, como a ferramenta computacional, permitiu uma maior participação dos alunos nas aulas, havendo uma troca de informação satisfatória para os envolvidos.

\section{Conclusão}

Conclui-se que é muito importante que alunos saibam do amplo campo de aplicação que conteúdos teóricos vistos em sala possuem e, por meio de ferramentas tecnológicas, essa ideia pode ser explorada, contribuindo com uma maior assimilação de conteúdos estudados. Ao decorrer do desenvolvimento do projeto, pode-se tornar menos abstrato aos discentes um conteúdo que, normalmente, somente é visto no ensino superior, sendo um ponto positivo caso estes mesmos alunos venham a rever o conteúdo futuramente.

\section{Agradecimentos}

Os autores agradecem a FAPES pelo apoio financeiro e a UFES por ceder os laboratórios computacionais para que o projeto pudesse ser realizado.

\section{Referências}

[1] SANTOS, R. P. DOS; COSTA, H. A. X. TBC-AED e TBC-AED/Web: Um desafio no ensino de algoritmos, estruturas de dados e programação. In: IV Workshop Em Educação Em Computação E Informática Do Estado De Minas Gerais. 2005.

[2] ROCHA, P. S. et al. Ensino e aprendizagem de programação: análise da aplicação de proposta metodológica baseada no sistema personalizado de ensino. RENOTE, v. 8, n. 3, 2010.

[3] RAABE, A. L. A.; SILVA, J. M. C. 2005 . Um Ambiente para Atendimento às Dificuldades de Aprendizagem de Algoritmos." In: XIII Workshop sobre Educação em Computação, São Leopoldo/RS.

[4] MEC. 1998. Ministério da Educação. Conselho Nacional de Educação. Diretrizes Curriculares Nacionais para o Ensino Médio - Resolução CEB/CNE № 3, de 26 de junho de 1998.

[5] DIM, C. A.; ROCHA, F. E. L. APIN: Uma Ferramenta Para Aprendizagem de Lógicas e Estímulo do Raciocínio e da Habilidade de Resolução de Problemas em um Contexto Computacional no Ensino Médio. In: XXI Congresso da Sociedade Brasileira de Computação, 2011, Natal. XXI Congresso da Sociedade Brasileira de Computação, 2011. 